\title{
Comparison Principles and Pointwise Estimates for Viscosity Solutions of Nonlinear Elliptic Equations
}

Neil S. Trudinger

\begin{abstract}
We prove comparison principles for viscosity solutions of nonlinear second order, uniformly elliptic equations, which extend previous results of P.L. Lions, R. Jensen and H. Ishii. Some basic pointwise estimates for classical solutions are also extended to continuous viscosity solutions.
\end{abstract}

\section{Introduction}

In this paper we are concerned with some comparison principles and local estimates for viscosity subsolutions and supersolutions of nonlinear uniformly elliptic differential equations of the form,

$$
F[u]=F\left(x, u, D u, D^{2} u\right)=0
$$

in domains $\Omega$ in Euclidean $n$-space, $\mathbb{R}^{n}$. Here the function $F \in C^{0}(\Gamma)$ where $\Gamma=\Omega \times \mathbb{R} \times \mathbb{R}^{n} \times \mathbb{S}^{n}, \mathbb{S}^{n}$ denotes the linear space of real $n \times n$ symmetric 
matrices and the operator $F$ is at least degenerate elliptic, that is,

$$
F(x, z, p, r) \leqslant F(x, z, p, r+\eta)
$$

for all $x, z, p, r \in \Gamma, \eta \geqslant 0, \eta \in \mathbb{S}^{n}$. Following Lions [16], a function $u \in C^{0}(\Omega)$ is called a viscosity subsolution (supersolution) of equation (1.1) if for any function $\varphi \in C^{2}(\Omega)$ and local maximum (minimum) $x_{0}$ of $u-\varphi$, we have

$$
F\left(x_{0}, u\left(x_{0}\right), D \varphi\left(x_{0}\right), D^{2} \varphi\left(x_{0}\right)\right) \geqslant 0, \quad(\leqslant 0) .
$$

A viscosity solution is both subsolution and supersolution. Such a notion of solution is implicit in the classical Perron process but was invoked recently by Crandall and Lions [4] in the context of first order equations. Lions [16] treated the existence and uniqueness of viscosity solutions of second order equations in the case that (1.1) is a Bellman equation, arising from stochastic control theory. The first significant breakthrough in the general case was made by Jensen [9], who established comparison principles for Lipschitz solutions when the function $F$ is essentially independent of the $x$ variables. His result was extended to continuous solutions by Jensen, Lions and Souganidis [11] and the latter restriction removed independently by Ishii [7], Jensen himself [10] and the present author (for uniformly elliptic operators) using different methods. The main point of this paper is to demonstrate our approach which employs a combination of Jensen's ideas in [9] and a second derivative estimate for linear equations, due to Lin [15]. By adjoining the technique, used by Ishii [7], of adding more variables we are able to improve the results of Ishii and Jensen in the uniformly elliptic case. This latter technique was also utilized independently by us for regularity considerations [18], [19] and in this context it arose from a classical regularity argument which we supplied in [14, Theorem 1].

In order to describe our comparison principles we formulate structure conditions as follows:

\section{F1 (Uniform ellipticity)}

$$
\lambda \text { trace }(\eta) \leqslant F(x, z, p, r+\eta)-F(x, z, p, r) \leqslant \Lambda \text { trace }(\eta) ;
$$

\section{F2 (Lipschitz-continuity in $p$ )}

$$
|F(x, z, p, r)-F(x, z, q, r)| \leqslant \mu_{1}|p-q| ;
$$

\section{F3 (Monotonicity in z)}

$$
F(x, z, p, r) \leqslant F(x, z+t, p, r) ;
$$

F4 (Continuity in $x$ )

$$
|F(x, z, p, r)-F(y, z, p, r)| \leqslant \mu_{2}|x-y|^{\delta}|r|+\omega(|x-y|) ;
$$


for all $x, y \in \Omega,|z| \leqslant K,|p|,|q| \leqslant K_{1}, r \in \mathbb{S}^{n}, \eta \geqslant 0, \eta \in \mathbb{S}^{n}, t, K, K_{1} \in \mathbb{R}^{+}$, where $\lambda, \Lambda, \mu_{1}, \mu_{2}, \delta$ are fixed positive constants (depending possibly on $K$ ) and $\omega(a) \rightarrow 0$ as $a \rightarrow 0$.

We can then state the following comparison principle.

Theorem 1.1. Let $u, v \in C^{0}(\bar{\Omega}) \cap C^{0,1}(\Omega)$ be respectively subsolution and supersolution of equation (1.1) in $\Omega$, with $u \leqslant v$ on $\partial \Omega$. Then if the structure conditions $F 1, F 2, F 3, F 4$ are satisfied for $\delta=1 / 2$, we have $u \leqslant v$ in $\Omega$.

In fact more general results hold. Namely one can allow $\delta<1 / 2$, depending on $n, \Lambda / \lambda$, and if only $u$ or $v \in C^{0}(\bar{\Omega}) \cap C^{0, \gamma}(\Omega), 0 \leqslant \gamma \leqslant 1$, (with $F 1-F 4$ holding for all $p, q \in \mathbb{R}^{n}$ ), the conclusion holds for some $\delta<1-\gamma / 2$. In the work of Jensen [10] and Ishii and Lions [8], which extends Ishii [7], the second inequality in $F 1$ can be dispensed with but one must have $\delta>1 / 2$. The further relaxation of $F 4$ seems to be an open problem. Of course if $u, v \in W^{2, n}(\Omega)$, no such condition is required as the usual comparison principle then holds, (for example see [6]). Theorem 1.1 implies a uniqueness result for the Dirichlet problem, previously announced by us in [18, Remark 3.3].

The plan of this paper is as follows. In the following section we develop some auxiliary results for semi-convex subsolutions of linear eliptic equations. These are applied to yield comparison principles in Section 3, initially in the case $\delta=1$ which we originally obtained. As consequences of Theorem 1.1 we obtain a strong comparison principle and a comparison principle for oblique boundary conditions. The results of Section 3 are extended to Hölder continuous solutions in Section 4. In Section 5 we note some of the pointwise estimates which also arise from the estimates of Section 2. In a further work [20] we show that viscosity solutions of uniformly elliptic equations are twice differentiable almost everywhere. Unless otherwise stated all notation in this paper follows the book [6].

The author is grateful to the Max Planck Institut für Mathematik in Bonn where the research for this paper was completed in 1987, and to P.L. Lions for informing him of related work [7], [8], [11], after we had proved the case $\delta=1$ in Theorem 1.1.

\section{Auxiliary Results for Linear Equations}

Jensen's approach to viscosity solutions is based on their approximation by semi-convex subsolutions and semi-concave supersolutions. In this section we consider the appropriate extensions of the linear theory to such subsolutions and supersolutions. We first note that a function $u$ on the domain $\Omega$ is called semi-convex (semi-concave) if $u=v+w$ for some $v \in C^{1,1}(\Omega)$ and convex 
(concave) $w$. Such solutions are twice differentiable almost everywhere in $\Omega$. We consider linear elliptic operators of the form

$$
L u=a^{i j} D_{i j} u+b^{i} D_{i} u
$$

with coefficients $a^{i j}, b^{i}, i, j=1, \ldots, n$ satisfying

$$
\lambda I \leqslant\left[a^{i j}\right]<\Lambda I, \quad\left|b^{i}\right| \leqslant \mu,
$$

for positive constants $\lambda, \Lambda, \mu$.

Lemma 2.1. Let $u$ be semi-convex in $\Omega$ and suppose $L u+f \geqslant 0$ almost everywhere in $\Omega$ for some $f \in L^{n}(\Omega)$. We then have the following estimates:

I. (Aleksandrov maximum principle.)

$$
\sup _{\Omega} u \leqslant \sup _{\partial \Omega} u+C \operatorname{diam} \Omega\left\|f / D^{*}\right\|_{L^{n}\left(\Gamma^{+}\right)},
$$

where $C$ depends on $n,\left\|b / D^{*}\right\|_{n}$ and $D^{*}=\left(\operatorname{det}\left[a^{i j}\right]\right)^{1 / n}, \Gamma^{+}$is the upper contact set of $u$ (see [6]);

II. (Local maximum principle.) For any ball $B=B_{R}(y) \subset \Omega$, concentric subball $B_{\sigma}=B_{\sigma R}(y), 0<\sigma<1$, and $p>0$,

$$
\sup _{B_{\sigma}} u \leqslant C\left\{\left(R^{-n} \int_{B}\left(u^{+}\right)^{p}\right)^{1 / p}+R\|f / \lambda\|_{L^{n(B)}}\right\},
$$

where $C$ depends on $n, \Lambda / \lambda, \mu R / \lambda, \sigma$ and $p$;

III. (Weak Harnack inequality.) For any ball $B=B_{R}(y) \subset \Omega$ and concentric subball $B_{\sigma}, 0<\sigma<1$, there exists a positive number $p$, depending only on $n$, $\Lambda / \lambda$, such that

$$
\left(R^{-n} \int_{B_{\sigma R}}(M-u)^{p}\right)^{p}<C\left\{\inf _{B_{\sigma R}}(M-u)+R\|f / \lambda\|_{L^{n}(B)}\right\}
$$

where $M=\sup _{B_{R}} u$ and $C$ depends on $n, \Lambda / \lambda, \mu R / \lambda$ and $\sigma$.

IV. (Derivative estimates.) There exists a positive number $\varkappa$, depending only on $n, \Lambda / \lambda$, such that, for any subdomain $\Omega^{\prime} \subset \subset \Omega$,

$$
\left(\int_{\Omega^{\prime}}|D u|^{\varkappa}+\left|D^{2} u\right|^{\varkappa}\right)^{1 / \varkappa} \leqslant C\left\{\sup _{\Omega}|u|+\|f / \lambda\|_{L^{n(\Omega)}}\right\}
$$

where $C$ depends on $n, \Lambda / \lambda, \mu / \lambda$, $\operatorname{diam} \Omega$, dist $\left(\Omega^{\prime}, \partial \Omega\right)$. 
The estimates I, II, III are established in the literature for subsolutions $u \in W^{2, n}(\Omega)$; (see for example [2], [6], [17]). The extension of the Aleksandrov estimate (2.3) follows automatically from his work [2] since the normal mapping of semi-convex functions will be absolutely continuous with respect to Lebesgue measure. Alternatively, all of the above estimates may be deduced, from their validity for classical subsolutions, by mollification since the Hessian matrices of the mollifications of a semi-concave function will be uniformly bounded from below.

The estimate IV was proved by Evans [5] and Lin [15], for the first and second derivatives respectively of solutions of the Dirichlet problem. To obtain a local estimate for subsolutions $u \in C^{2}(\Omega)$ we may first observe from Lin [15] that there exists a positive matrix function $\left[a_{*}^{i j}\right]$, depending on $D^{2} u$, such that

$$
\begin{gathered}
\frac{\lambda}{2}\left|D^{2} u\right| \leqslant\left(a_{*}^{i j}-a^{i j}\right) D_{i j} u, \\
\frac{\lambda}{2} I \leqslant\left[a_{*}^{i j}\right] \leqslant 2 \Lambda I .
\end{gathered}
$$

For $\eta \in C_{0}^{2}(\Omega), k<\inf u$ and

$$
v=\eta^{2}(u-k)^{2}
$$

we now compute

$$
\begin{aligned}
a_{*}^{i j} D_{i j} v= & 2 \eta^{2}(u-k) a_{*}^{i j} D_{i j} u+2 \eta^{2} a_{*}^{i j} D_{i} u D_{j} u+8 \eta(u-k) a_{*}^{i j} D_{i} \eta D_{j} u \\
& +(u-k)^{2} a_{*}^{i j} D_{i j} \eta^{2} \\
\geqslant & \frac{\lambda}{2} \eta^{2}\left\{(u-k)\left|D^{2} u\right|+|D u|^{2}\right\} \\
& -C(u-k)\left\{\eta^{2}(u-k)|b|^{2}+\eta^{2}|f|+\Lambda(u-k)\left(|D \eta|^{2}+\left|\eta D^{2} \eta\right|\right\}\right. \\
= & \frac{\lambda}{2} \eta^{2}\left\{(u-k)\left|D^{2} u\right|+|D u|^{2}\right\}-f_{*}
\end{aligned}
$$

for some constant $C$. It then follows from Evans [5] (or Lin [15]) that there exists some positive $\varkappa$ depending only on $n, \Lambda / \lambda$ for which

$$
\left\{\int_{\Omega} \eta^{2 x}\left[(u-k)\left|D^{2} u\right|+|D u|^{2}\right]^{x}\right\}^{1 / x} \leqslant C\left\|f_{*} / \lambda\right\|_{L^{n(\Omega)}}
$$

where $C$ depends on $n, \Lambda / \lambda$ and diam $\Omega$. By appropriate choice of $\eta$ and $k$ we thus obtain the estimate (2.6). 


\section{Comparison Principles for Lipschitz Solutions}

In this section we prove Theorem 1.1, along with some applications. Following Jensen [9], we can approximate functions $u \in C^{0,1}(\Omega)$ by setting, for $\epsilon>0$,

$$
\Omega_{\epsilon}=\{x \in \Omega \mid \operatorname{dist}(x, \partial \Omega)>\epsilon\},
$$

and letting $u_{\epsilon}^{ \pm} \in C^{0,1}\left(\Omega_{\epsilon}\right)$ be the functions whose graphs have fixed distance $\epsilon$ from the graph of $u$ and which lie respectively above and below the graph of $u$. The graphs of $u_{\epsilon}^{ \pm}$will satisfy lower and upper exterior sphere conditions respectively, with spheres of radius $\epsilon$, and therefore, since the functions $u_{\epsilon}^{ \pm}$ are Lipschitz, they will be semi-convex and semi-concave respectively. Moreover we have the estimates

$$
\left|D u_{\epsilon}^{ \pm}\right| \leqslant|D u|_{0}
$$

and

$$
\pm D^{2} u_{\epsilon}^{ \pm} \geqslant-\frac{\left(1+|D u|_{0}^{2}\right)^{1 / 2}}{\epsilon}
$$

in the sense of distributions. A simple geometric consideration (together with condition $F 3$ ) shows that if $u$ is a viscosity subsolution (supersolution) of equation (1.1) in $\Omega$, then the functions $u_{\epsilon}^{+}-\epsilon\left(u_{\epsilon}^{+}+\epsilon\right)$ will be a viscosity subsolution (supersolution) with the operator $F$ replaced by $F^{+}\left(F^{-}\right)$given by

$$
F^{ \pm}(x, z, p, r)=F\left(x^{ \pm}, z, p, r\right),
$$

where

$$
x^{ \pm}=x \pm \epsilon \nu^{ \pm}, \quad \nu^{ \pm}=\frac{D u_{\epsilon}^{ \pm}}{\sqrt{1+\left|D u_{\epsilon}^{ \pm}\right|^{2}}}
$$

is the projection in $\mathbb{R}^{n}$ of the unit normal to the graph of $u_{\epsilon}^{ \pm}$. Accordingly, (replacing $u_{\epsilon}^{ \pm}$by $u_{\epsilon}^{ \pm} \mp \epsilon$ ), we have (see [9], [18], [7]) the differential inequalities,

$$
\pm F^{ \pm}\left[x, u_{\epsilon}^{ \pm}, D u_{\epsilon}^{ \pm}, D^{2} u_{\epsilon}^{ \pm}\right] \geqslant 0
$$

almost everywhere in $\Omega_{\epsilon}$.

We first prove Theorem 1.1 for the case $\delta=1$, which was our original result. If $u, v$ satisfy the hypotheses of Theorem 1.1, we have (almost everywhere) in the set where

$$
w_{\epsilon} \equiv u_{\epsilon}^{+}-v_{\epsilon}^{-} \geqslant 0
$$


the differential inequality,

(3.5) $L w_{\epsilon} \equiv a^{i j} D_{i j} w_{\epsilon}+b^{i} D_{i} w_{\epsilon}$

$$
\begin{aligned}
& \geqslant F\left(x^{-}, v_{\epsilon}^{-}(x), D u_{\epsilon}^{+}(x), D^{2} u_{\epsilon}^{+}(x)\right)-F\left(x^{+}, v_{\epsilon}^{-}(x), D u_{\epsilon}^{+}(x), D^{2} u_{\epsilon}^{+}(x)\right) \\
& \geqslant-2 \mu_{2} \epsilon\left|D_{2} u_{\epsilon}^{+}\right|-\omega(2 \epsilon),
\end{aligned}
$$

by $F 4$, with coefficients $a^{i j}, b^{i}$ given by

$$
\begin{aligned}
a^{i j} & =\int_{0}^{1} F_{r_{i j}}^{-}\left(x, v_{\epsilon}^{-}, D v_{\epsilon}^{-}, D^{2}\left(v_{\epsilon}^{-}+t w_{\epsilon}\right)\right) d t, \\
b^{i} & =\int_{0}^{1} F_{p_{i}}^{-}\left(x, v_{\epsilon}^{-}, D\left(v_{\epsilon}^{-}+t w_{\epsilon}\right), D^{2} u_{\epsilon}^{+}\right) d t .
\end{aligned}
$$

Using the structure conditions $F 1, F 2$ we see that

$$
\begin{aligned}
& \lambda I \leqslant\left[a^{i j}\right] \leqslant \Lambda I, \\
& |b| \leqslant \mu_{1} .
\end{aligned}
$$

If the constant $\mu_{2}=0$, then Theorem 1.1 follows immediately from the Aleksandrov maximum principle and we have as a special case Jensen's result [9]. Here of course there is no need for the upper bound in (3.7) and the result is readily extended to the case of degenerate ellipticity and a strict monotonicity condition $F 2$. To handle the second derivative term on the right hand side of (3.5), we now write the differential inequality (3.3) for $u_{\epsilon}^{+}$in the form,

$$
\begin{aligned}
L_{0} u_{\epsilon}^{+} & \equiv a_{0}^{i j} D_{i j} u_{\epsilon}^{+}+b_{0}^{i} D_{i} u_{\epsilon}^{+} \\
& \geqslant-F^{+}\left(x, u_{\epsilon}^{+}-\epsilon, 0,0\right) \\
& \geqslant-F_{0}
\end{aligned}
$$

for some constant $F_{0}$, where the coefficients $a_{0}^{i j}, b_{0}^{i}$, given by

$$
\begin{aligned}
a_{0}^{i j} & =\int_{0}^{1} F_{r_{i j}}^{+}\left(x, u_{\epsilon}^{+}, D u_{\epsilon}^{+}, t D^{2} u_{\epsilon}^{+}\right) d t, \\
b_{0}^{i} & =\int_{0}^{1} F_{p_{i}}^{+}\left(x, u_{\epsilon}^{+}, t D u_{\epsilon}^{+}, 0\right) d t,
\end{aligned}
$$

also satisfy (3.7). From the second derivative estimate, Lemma 2.1, IV, we then have

$$
\int_{\Omega^{\prime}}\left|D^{2} u_{\epsilon}^{+}\right|^{x} \leqslant C
$$

for any $\Omega^{\prime} \subset \subset \Omega$, where $\varkappa$ depends only on $n, \Lambda / \lambda$ and $C$ is independent of $\epsilon$. Fixing $\Omega^{\prime} \subset \subset \Omega_{\epsilon}$ we now apply the Aleksandrov maximum principle, Lemma $2.1, \mathrm{I}$, to the function $w_{\epsilon}$, noting that on the upper contact set $\Gamma^{+}$of $w_{\epsilon}$, we have by virtue of (3.1),

$$
\left|D^{2} u_{\epsilon}^{+}\right| \leqslant \frac{C}{\epsilon} .
$$


Accordingly, we obtain

$$
\begin{aligned}
\sup _{\Omega^{\prime}} w_{\epsilon} & \leqslant C\left\{\epsilon\left\|D^{2} u_{\epsilon}^{+}\right\|_{L^{n}\left(\Gamma^{+}\right)}+\omega(2 \epsilon)\right\}+\sup _{\partial \Omega^{\prime}} w_{\epsilon}^{+} \\
& \leqslant C\left\{\epsilon^{\varkappa / n}+\omega(2 \epsilon)\right\}+\sup _{\partial \Omega^{\prime}} w_{\epsilon}^{+}, \text {by } \quad(3.10) \\
& \rightarrow \sup _{\partial \Omega^{\prime}} w_{\epsilon}^{+}, \text {as } \epsilon \rightarrow 0, \\
& \rightarrow 0 \text { as } \quad \Omega^{\prime} \rightarrow \Omega .
\end{aligned}
$$

Consequently $u \leqslant v$ in $\Omega$ and Theorem 1.1 is proved in the case $\delta=1$.

To treat the general case, we extend $w$ as a function of $2 n$ variables $(x, \xi)$ on the domain,

$$
\bar{\Omega}=\Omega_{2 \epsilon} \times B_{0}(1) \subset \mathbb{R}^{2 n},
$$

by defining

$$
w_{\epsilon}(x, \xi)=u_{\epsilon}^{+}(x)-v_{\epsilon}^{-}(x+\epsilon \xi) .
$$

We then obtain (instead of 3.5),

$$
L w_{\epsilon} \geqslant-\mu_{2}(3 \epsilon)^{\delta}\left|D^{2} u_{\epsilon}^{+}\right|-\omega(3 \epsilon),
$$

where in the formulae (3.6) for the coefficients $a^{i j}, b^{i}$, the arguments of $v_{\epsilon}^{-}$ are $x+\epsilon \xi$ instead of $x$. Writing

$$
g=\left|D^{2} u_{\epsilon}^{+}\right|^{x / 2 n},
$$

we may then estimate

$$
L w_{\epsilon} \geqslant-C \epsilon^{\delta \chi} g\left|D^{2} u_{\epsilon}^{+}\right|-o(\epsilon)
$$

where $\chi=1+\varkappa / 2 n$ and $\delta$ is replaced by any smaller number. Now come some technical subtleties. Defining

$$
\sigma^{i j}=\left\{\begin{array}{ccc}
D_{i j} u_{\epsilon}^{+} /\left|D^{2} u_{\epsilon}^{+}\right| & \text {if } & D^{2} u_{\epsilon}^{+} \neq 0, \\
0 & \text { if } & D^{2} u_{\epsilon}^{+}=0,
\end{array}\right.
$$

we write (3.16) in the form

$$
\begin{aligned}
L w_{\epsilon} & \geqslant-C \epsilon^{\delta x} g \sigma^{i j} D_{i j} u_{\epsilon}^{+}-o(\epsilon) \\
& =-C \epsilon^{\delta x} g \sigma^{i j}\left(D_{i j} w+D_{i j} v_{\epsilon}^{-}\right)-o(\epsilon) \\
& =-C \epsilon^{\delta x} g \sigma^{i j}\left(D_{i j} w-\frac{1}{\epsilon} D_{i \xi_{j}} w\right)-o(\epsilon) .
\end{aligned}
$$


To proceed further we need to write (3.17) as an elliptic differential inequality in the variables $(x, \xi)$. First let us suppose that

$$
C \epsilon^{\delta x} g \leqslant \frac{\lambda}{3}
$$

to guarantee that inequality (3.17) is at least elliptic in the $x$ variables (with minimum eigenvalue $\geqslant 2 \lambda / 3$ ). Setting, analogously to (3.9),

$$
a_{0}^{i j} \equiv \int_{0}^{1} F_{r_{i j}}^{-}\left(x, v_{\epsilon}^{-}, D v_{\epsilon}^{-}, t D^{2} v_{\epsilon}^{-}\right) d t,
$$

we next have

$$
a_{0}^{i j} D_{\xi_{i} \xi_{j}} w \geqslant-\epsilon^{2} a_{0}^{i j} D^{i j} v_{\epsilon}^{-} \geqslant-C_{1} \epsilon^{2}
$$

for a further constant $C_{1}$. Consequently

$$
\begin{aligned}
\tilde{L} w_{\epsilon} & \equiv L w_{\epsilon}+C \epsilon^{\delta x} g \sigma^{i j}\left(D_{i j} w_{\epsilon}-\epsilon^{-1} D_{i \xi_{j}} w_{\epsilon}\right)+3 n\left(C g \epsilon^{\delta \chi-1} / \lambda\right)^{2} a_{0}^{i j} D_{\xi_{i} \xi_{j}} w_{\epsilon} \\
& \geqslant-C_{1} g^{2} \epsilon^{2 \delta \chi}-o(\epsilon)
\end{aligned}
$$

for constants $C, C_{1}$ independent of $\epsilon$, and the operator $\tilde{L}$ is elliptic if (3.18) is satisfied.

We next need to modify $w_{\epsilon}$ so that it cannot take its maximum for $|\xi|=1$. Using the Lipschitz continuity of $v$ and (3.1), we set

$$
\tilde{w}_{\epsilon}(x, \xi)=w_{\epsilon}(x, \xi)-2 K \epsilon|\xi|^{2},
$$

where $K=|D u|_{0}$, to guarantee

$$
\sup _{|\xi|=1} \tilde{w}_{\epsilon}(x, \xi) \geqslant \tilde{w}_{\epsilon}(x, 0)-K \epsilon .
$$

From (3.19) we then obtain

$$
\begin{aligned}
\tilde{L} \tilde{w}_{\epsilon} & \geqslant-C_{1} g^{2} \epsilon^{2 \delta x-1}-o(\epsilon) \\
& \equiv-\tilde{g}(\leqslant 0),
\end{aligned}
$$

for a further constant $C_{1}$, independent of $\epsilon$. The inequality (3.23) prevents us from directly applying Lemma 2.1 , as in the case $\delta=1$, so we first need to convert (3.24) to a homogeneous inequality. To achieve this we fix a subdomain $\Omega^{\prime} \subset \subset \Omega_{2 \epsilon}$ and a ball $\tilde{B} \subset \Omega$ and extend $\tilde{g}$ to vanish in $\tilde{B}-\Omega^{\prime}$. We then solve the following generalized Dirichlet problem for the Monge-Ampère equation,

$$
\begin{gathered}
\operatorname{det} D^{2} G=h(x, D G) \text { in } \tilde{B}, \\
G=0 \text { on } \partial \tilde{B},
\end{gathered}
$$


where

$$
h(x, p)=\left(\frac{2 n}{\lambda}\right)^{n}\left(|p|^{n /(n-1)}+g_{0}^{n /(n-1)}\right)^{n-1}\left(\mu^{n}+g_{0}^{-n}|\tilde{g}|^{n}\right),
$$

and

$$
g_{0}=\frac{2}{\lambda}\|\tilde{g}\|_{n}
$$

If $\mu=0$, we can simply take

$$
h(x, p)=\left(\frac{2 n \tilde{g}}{\lambda}\right)^{n} .
$$

From the theory of Aleksandrov [1] and Bakel'man [3], there exists a unique convex generalized solution $G$ satisfying (3.25) almost everywhere in $\Omega$. Furthermore, we can estimate, (see also [6, Section 9.1]),

$$
\begin{aligned}
\inf G & \geqslant-C\|\tilde{g}\|_{L^{n}\left(\Omega^{\prime}\right)} \\
& \geqslant-C \epsilon^{2 \delta x-1}-o(\epsilon),
\end{aligned}
$$

by virtue of (3.10), (3.15) and (3.24). Moreover, we now have

$$
\tilde{L}\left(\tilde{w}_{\epsilon}+G\right) \geqslant 0,
$$

almost everywhere on the subset of $\Omega^{\prime} \times B$ where $w_{\epsilon}>0$ and (3.18) holds. Since $G$ is convex the inequality (3.11) continues to hold on the upper contact set of the function $\tilde{w}_{\epsilon}+G$, whence condition (3.18) is fulfilled there for sufficiently small $\epsilon$. Applying the Aleksandrov maximum principle, Lemma 2.1, I, to the semi-convex function $\tilde{w}_{\epsilon}+G$, we find

$$
\begin{aligned}
\sup _{x \in \Omega^{\prime}}\left(w_{\epsilon}+G\right) & \leqslant \sup _{\partial\left(\Omega^{\prime} \times B\right)}\left(\tilde{w}_{\epsilon}+G\right)^{+}+o(\epsilon) \\
& \leqslant \sup _{\partial \Omega^{\prime} \times B} w_{\epsilon}^{+}+o(\epsilon),
\end{aligned}
$$

by (3.23), and hence we conclude, by (3.26),

$$
\begin{aligned}
\sup _{\Omega^{\prime}} w_{\epsilon} & \leqslant C \epsilon^{2 \delta x-1}+o(\epsilon)+\sup _{\partial \Omega^{\prime}} w_{\epsilon}^{+} \\
& \rightarrow \sup _{\partial \Omega^{\prime}}(u-v)^{+} \text {as } \quad \epsilon \rightarrow 0, \\
& \rightarrow \sup _{\partial \Omega}(u-v)^{+} \text {as } \quad \Omega^{\prime} \rightarrow \Omega,
\end{aligned}
$$


provided $2 \delta \chi-1>0$, that is

$$
\delta>\frac{1}{2+x / n} .
$$

We therefore have

$$
\sup _{\Omega}(u-v) \leqslant \sup _{\Omega}(u-v)^{+}
$$

and the proof of Theorem 1.1 is complete.

Remark 3.1. The continuity condition $F 4$ can be made more precise in terms of the value of $x$ in Lemma 2.1, IV. Namely, if we formulate $F 4$ as

$\tilde{F} 4:$

$$
|F(x, z, p, r)-F(y, z, p, r)| \leqslant \omega(|x-y|)\left(|x-y|^{\delta}|r|+1\right),
$$

then $\delta \geqslant 1 / 2(1+\varkappa / n)$ suffices for the validity of Theorem 1.1. If only the first inequality in $F 1$ holds, that is $F$ is strictly elliptic rather than uniformly elliptic, then we must have $\varkappa=0$, as in [10], and the proof of Theorem 1.1 simplifies greatly.

Remark 3.2. Following a standard argument, ([6, Section 3.2]), we can infer from the weak comparison principle, Theorem 1.1, a strong comparison principle and a boundary point inequality. Namely,

(i) (Strong comparison principle.) If $u$ and $v \in C^{0,1}(\Omega)$ are respectively subsolution and supersolution of equation (1.1) in $\Omega$, then the difference $w=u-v$ cannot take a positive maximum in $\Omega$ unless it is constant.

(ii) (Boundary point lemma.) If the above function $w$ assumes a strict positive maximum, continuously at a boundary point $x_{0}$, where $\partial \Omega$ satisfies an interior sphere condition (with sphere $S$ ) then

$$
D_{\beta}^{+} w\left(x_{0}\right)=\limsup _{t \rightarrow 0} \frac{w\left(x_{0}+t \beta\right)-w\left(x_{0}\right)}{t}<0
$$

for any $\beta \cdot \nu>0$ where $\nu$ is the inner normal to $S$ at $x_{0}$.

Remark 3.3. The previous remark yields comparison principles for oblique boundary conditions. Let $G$ be a continuous function on $\Gamma_{0}=\partial \Omega \times \mathbb{R} \times \mathbb{R}^{n}$ and suppose that an inner normal $\nu$ exists on $\partial \Omega$ in a reasonable sense, for example $\partial \Omega \in C^{1}$ (or more generally $\partial \Omega$ satisfies an interior cone condition). Then $G$ is degenerate oblique (oblique) if

$$
G(x, z, p+t \nu)-G(x, z, p) \geqslant 0 \quad(>0)
$$


for all $x, z, p \in \Gamma_{0}, t>0$. For $u \in C^{0}(\bar{\Omega})$, the boundary conditions $G[u] \geqslant 0$ $(\leqslant 0)$ are then satisfied in the viscosity sense if for any $\varphi \in C^{1}(\bar{\Omega})$ and local maximum (minimum) $x_{0} \in \partial \Omega$ of $u-\varphi$, we have

$$
G\left(x_{0}, u\left(x_{0}\right), D \varphi\left(x_{0}\right)\right) \geqslant 0 \quad(\leqslant 0) .
$$

We then have

Corollary 3.1. Let $u, v \in C^{0,1}(\bar{\Omega})$ be respectively subsolution and supersolution of equation (1.1) in $\Omega$ satisfying $G[u] \geqslant 0, G[v] \leqslant 0$ on $\partial \Omega$, where $\partial \Omega$ satisfies an interior sphere condition and $G$ is strictly oblique (with respect to an interior sphere normal at each boundary point) and non-increasing with respect to $z$. Then either $u \leqslant v$ in $\Omega$ or $u-v$ is a positive constant.

Proof. If $w=u-v$ takes a positive maximum at $x_{0} \in \partial \Omega$, so also does $w^{*}=$ $u^{*}-v^{*}$ where $u^{*}, v^{*} \in C^{1}(\bar{\Omega})$ satisfy $u^{*} \geqslant u, v^{*} \leqslant v$ in $\Omega$,

$$
u^{*}\left(x_{0}\right)=u\left(x_{0}\right), v^{*}\left(x_{0}\right)=v\left(x_{0}\right) .
$$

But then

$$
\begin{aligned}
G\left(x_{0}, v\left(x_{0}\right), D v^{*}\left(x_{0}\right)\right) & \leqslant 0 \\
& \leqslant G\left(x_{0}, u\left(x_{0}\right), D u^{*}\left(x_{0}\right)\right) \\
& \leqslant G\left(x_{0}, v\left(x_{0}\right), D v^{*}\left(x_{0}\right)+\nu \cdot D w^{*}\left(x_{0}\right)\right)
\end{aligned}
$$

which contradicts (3.35) since $D_{\nu} w^{*}\left(x_{0}\right)$ can be chosen arbitrarily close to $D_{\nu}^{+} w$ for $u, v \in C^{0,1}(\bar{\Omega})$.

Remark 3.4. Finally we remark that if $u$ or $v \in C^{1, \gamma}(\Omega), \gamma>0$, then by replacing $\epsilon$ by $\epsilon^{1+\gamma}$ in the approximations $u_{\epsilon}^{ \pm}$and (3.22) with $K=2[D u]_{\gamma}$, we obtain the above results for

$$
\delta>(1-\gamma) /(2+\varkappa / n) .
$$

If $u$ or $v \in C^{1}(\Omega)$, the proof of Theorem 1.1 can be effected with $\varkappa=0$.

\section{Comparison Principles for Hölder Solutions}

In this section we extend Theorem 1.1 to embrace solutions $u, v \in C^{0, \alpha}(\Omega)$ where possibly $\alpha \leqslant 1$. The reduced smoothness of $u, v$ must be compensated by a strengthening of condition $F 4$ and we must take $K_{1}=\infty$ in conditions $F 1$ to $F 4$. We shall in fact modify $F 4$ to extend $\tilde{F} 4$ as follows:

$F^{*} 4: \quad|F(x, z, p, r)-F(y, z, p, r)| \leqslant \omega(|x-y|)\left(|x-y|^{\delta}|r|+|x-y|^{\delta *}|p|+1\right)$ 
where $\delta, \delta^{*}$ are non-negative constants to be specified. Throughout this section, unless otherwise stated, we assume $F 1, F 2, F 3, F^{*} 4$ to hold for all $x, y \in \Omega,|z| \leqslant K, p, q \in \mathbb{R}^{n}, r, \eta \geqslant 0, \eta \in \mathbb{S}^{n}, t, K \in \mathbb{R}^{+}$. We then have the following extension of Theorem 1.1.

Theorem 4.1. Let $u, v \in C^{0}(\bar{\Omega}) \cap C^{0, \alpha}(\Omega), 0 \leqslant \alpha \leqslant 1$, be respectively subsolution and supersolution of equation (1.1) in $\Omega$, with $u \leqslant v$ on $\partial \Omega$. Then if the structure conditions $F 1, F 2, F 3, F^{*} 4$ are satisfied for

$$
\delta \geqslant(1-\alpha / 2) /(1+\varkappa / n), \quad \delta^{*} \geqslant(1-\alpha) /(1+\varkappa / n),
$$

we have $u \leqslant v$ in $\Omega$.

Proof. Although the approximations $u_{\epsilon}^{+}, v_{\epsilon}^{-}$are now not necessarily semiconvex, semi-concave, they will be in the upper contact set of the function $w_{\epsilon}$ in the proof of Theorem 1.1 and consequently the case when $\varkappa=0(g \equiv 1)$ follows by only minor adjustment. As there appears some difficulty in extending the Lin estimate (3.10), we shall employ instead the corresponding approximations of Jensen, Lions and Souganidis [11] which are semi-convex, semi-concave everywhere. Accordingly, let us define for positive $\epsilon$, the functions

$$
\begin{aligned}
& u_{\epsilon}^{+}(x)=\sup _{y \in \Omega}\left\{u(y)-\omega_{0} \frac{|x-y|^{2}}{\epsilon^{2}}\right\}, \\
& u_{\epsilon}^{-}(x)=\inf _{y \in \Omega}\left\{u(y)+\omega_{0} \frac{|x-y|^{2}}{\epsilon^{2}}\right\}
\end{aligned}
$$

where $\omega_{0}=\operatorname{osc}_{\Omega} u$. It is clear that the supremum and infimum will be achieved in (4.2) at points $x^{ \pm}$satisfying $\left|x-x^{ \pm}\right|<\epsilon$ provided $x \in \Omega_{\epsilon}$ and moreover

$$
\begin{gathered}
\left|D u_{\epsilon}^{ \pm}\right| \leqslant \frac{2 \omega_{0}}{\epsilon}, \\
\pm D^{2} u_{\epsilon}^{+} \geqslant-\frac{2 \omega_{0}}{\epsilon^{2}}
\end{gathered}
$$

in the sense of distributions. Furthermore it is easily seen that if $u$ is a viscosity subsolution (supersolution) of equation (1.1) in $\Omega$, then $u_{\epsilon}^{+}\left(u_{\epsilon}^{-}\right)$will be a viscosity subsolution (supersolution) with the operator $F$ replaced by

$$
F^{ \pm}(x, z, p, r)=F^{ \pm}\left(x^{ \pm}, z, p, r\right) .
$$

We can now proceed as in the proof of Theorem 1.1 The dependence on $p$ in condition $F^{*} 4$ is handled by taking $g=\left(\left|D^{2} u\right|+|D u|\right)^{x / 2 n}$ and augmenting $\tilde{L}$ by terms in $D_{\xi} w_{\epsilon}$. Instead of (3.22) we take 


$$
\tilde{w}_{\epsilon}(x, \xi)=w_{\epsilon}(x, \xi)-2 \sigma(\epsilon)|\xi|^{2}
$$

where

$$
\sigma(\epsilon)=\sup _{y \in \Omega^{\prime}}\left(\underset{B_{2 \epsilon}(y)}{\operatorname{osc} u}\right) \leqslant C \epsilon^{\alpha} .
$$

Further details are left to the reader.

Remark 4.1. The case $\alpha=0$ was proved by Ishii [7] for subsolutions and supersolutions which need only be respectively upper-semicontinuous and lower-semicontinuous. A similar generalization holds here in that the conditions $u, v \in C^{0, \alpha}(\Omega)$ need only hold in a corresponding one-sided sense. Furthermore only $u$ or $v$ need lie in $C^{0, \alpha}(\Omega)$. The case $\varkappa=0$ is also proved in [10], but by different methods and, as we pointed out previously, only strict ellipticity (or degenerate ellipticity and strict monotonicity in $z$ ) suffices in condition $F 1$.

\section{Pointwise Estimates}

Using the approximations $u_{\epsilon}^{ \pm}$we can readily convert pointwise estimates for semi-convex subsolutions and semi-concave supersolutions to viscosity subsolutions and supersolutions. Consequently pointwise estimates such as the Hölder and Harnack estimates of Krylov and Safonov [13] can be extended to continuous viscosity solutions. Let us suppose that $F$ is uniformly elliptic, satisfying $F 1$ (with $K_{1}=\infty$ ) and

F5:

$$
|F(x, z, p, 0)|<\mu_{0}+\mu_{2}|p|^{2}
$$

for all $x \in \Omega,|z| \leqslant K, p \in \mathbb{R}^{n}$, where $\mu_{0}, \mu_{2}$ are further constants, depending on $K$. If $u$ is a subsolution of equation (1.1) then the functions

$$
v_{\epsilon}^{+}=\frac{1}{\mu_{2}}\left(e^{\mu_{2} u_{\epsilon}^{+}}-1\right)
$$

satisfy

$$
L_{0} v_{\epsilon}^{+} \equiv a_{0}^{i j} D_{i j} v_{\epsilon}^{+} \geqslant-\mu_{0} e^{\mu_{2}|u|_{0}}
$$

almost everywhere in $\Omega$, for some $\left[a^{i j}\right]$ satisfying (2.2) and we can apply, for example, the estimates in Section 2. In particular we obtain the local estimates. 
Theorem 5.1. Let $u \in C^{0}(\Omega), F$ satisfy $F 1, F 5$, and

$$
\begin{aligned}
B & =B_{R}(y) \subset \Omega, \\
B_{\sigma} & =B_{\sigma R}(y), \quad 0<\sigma<1 .
\end{aligned}
$$

I. (Local maximum principle.) If $u$ is a viscosity subsolution of (1.1),

$$
\sup _{B_{\sigma}} u \leqslant C\left\{\left(R^{-n} \int_{B}\left(u^{+}\right)^{p}\right)^{1 / p}+\mu_{0} R^{2} / \lambda\right\},
$$

for any $p>0$, where $C$ depends on $n, \Lambda / \lambda, \mu_{2}|u|_{0} / \lambda, \sigma$ and $p$;

II. (Weak Harnack inequality.) If $u$ is a non-negative supersolution of (1.1), there exists a constant $p$, depending on $n, \Lambda / \lambda$ such that

$$
\left(R^{-n} \int_{B_{\sigma}} u^{p}\right)^{1 / p}<C\left\{\inf _{B_{\sigma}} u+\mu_{0} R^{2} / \lambda\right\}
$$

where $C$ depends on $n, \Lambda / \lambda, \mu_{2}|u|_{0} / \lambda$ and $\sigma$.

III. (Hölder estimate.) If $u$ is a viscosity solution, then $u \in C^{\alpha}(\Omega)$ for some $\alpha>0$, depending on $n, \Lambda / \lambda$ and $\mu_{2}|u|_{0} / \lambda$ and

$$
\underset{B_{\sigma R}}{\operatorname{osc}} u \leqslant C \sigma^{\alpha}\left\{\begin{array}{l}
\operatorname{osc} u+\mu_{0} R^{2} / \lambda \\
B_{R}
\end{array}\right\}
$$

where $C$ also depends on $n, \Lambda / \lambda$ and $\mu_{2}|u|_{0} / \lambda$.

IV. (Harnack inequality.) If $u$ is a non-negative solution, then

$$
\sup _{B_{\sigma}} u \leqslant C\left\{\inf _{B_{\sigma}} u+\mu_{0} R^{2} / \lambda\right\},
$$

where $C$ depends on $n, \Lambda / \lambda$ and $\mu_{2}|u|_{0} / \lambda$.

Remark 5.1. Boundary gradient estimates for nonlinear elliptic equations may be extended in a similar fashion. In particular if $u \in C^{0}(\bar{\Omega}), \partial \Omega \in C^{1, \gamma}$, $u=g$ on $\partial \Omega, g \in C^{1, \gamma}(\bar{\Omega})$ for some $\gamma>0$ and $u$ is a viscosity solution of (1.1) in $\Omega$, then $u \in C^{0,1}(\bar{\Omega})$ and $v=(u-g) / \operatorname{dist}(x, \partial \Omega) \in C^{\alpha}(\bar{\Omega})$ for some $\alpha>0$ depending on $n, \Lambda / \lambda, \mu_{2}|u|_{0} / \lambda$ and $\gamma$, (see [6], [12]). Further regularity properties of viscosity solutions are treated in [18], [19], [20].

Remark 5.2. After completion of this manuscript, we received work by Caffarelli [21] on regularity, which overlaps [18], [19] and also proves III and IV above. 


\section{References}

[1] Aleksandrov, A. D. Dirichlet's problem for the equation det $\left\|Z_{i j}\right\|=\phi$, Vestnik Leningrad Univ. 13(1958), 5-24.

[2] Aleksandrov, A.D. Uniqueness conditions and estimates for solutions of the Dirichlet problem, Vestnik Leingrad Univ. 18(1963), 5-29; Amer. Math. Soc. Transl. (2) 68(1968), 89-119.

[3] Bakel'man, I. Ya. The Dirichlet problem for equations of Monge-Ampère type and their $n$ dimensional analogues, Dokl. Akad. Nauk SSSR 126(1959), 923-926.

[4] Crandall, M. G. and Lions, P.L. Viscosity solutions of Hamilton-Jacobi equations, Trans. Amer. Math. Soc. 277(1983), 1-42.

[5] Evans, L. C. Some estimates for non-divergence structure, second order elliptic equations. Trans. Amer. Math. Soc. 287(1985), 701-712.

[6] Gilbarg, D. and Trudinger, N.S. Elliptic partial differential equations of second order, 2nd edition, Springer-Verlag, 1983.

[7] Ishii, H. On uniqueness and existence of viscosity solutions of fully nonlinear second order elliptic PDE's, Comm. Pure Appl. Math. 42(1989), 15-45.

[8] Ishii, H. and Lions, P.L. Viscosity solutions of fully nonlinear second order elliptic partial differential equations, to appear.

[9] Jensen, R. The maximum principle for viscosity solutions of fully nonlinear second order partial differential equations, Arch. Rational Mech. Anal., 101(1988), 1-27.

[10] Jensen, R. Uniqueness criteria for viscosity solutions of fully nonlinear elliptic partial differential equations. Indiana Math. J., to appear.

[11] Jensen, R., Lions, P.L. and Souganidis, P.E. A uniqueness result for viscosity solutions of fully nonlinear second order partial differential equations, Proc. Amer. Math. Soc, 102(1988), 975-978.

[12] Krylov, N. V. Nonlinear elliptic and parabolic equations of the second order, Reidel, Dordrecht (1987).

[13] Krylov, N. V. and Safonov, M. V. Certain properties of parabolic equations with measurable coefficients, Izv. Akad. Nauk SSSR Ser. Mat. 40(1980), 161-175; Math. USSR. Izv. 16(1981), 151-164.

[14] Lieberman, G. M. Global regularity of solutions of nonlinear second order elliptic and parabolic differential equations. Math. Zeit. 193(1986), 331-346.

[15] Lin, F. H. Second derivative $L^{p}$ estimates for elliptic equations of nondivergent type. Proc. Amer. Math. Soc. 96(1986), 447-451.

[16] Lions, P.L. Optimal control of diffusion processes and Hamilton-JacobiBellman equations II, Comm. P.D.E. 8(1983), 1229-1276.

[17] Trudinger, N.S. Local estimates for subsolutions and supersolutions of general second order eliptic quasilinear equations, Invent. Math. 61(1980), 67-79.

[18] Trudinger, N.S. Hölder gradient estimates for fully nonlinear elliptic equations, Proc. Roy. Soc. Edinburgh, 108(1988), 57-65.

[19] Trudinger, N. S. On regularity and existence of viscosity solutions of nonlinear second order elliptic equations, Partial differential equations and the calculus of variations. Birkhouser (1989), 939-957.

[20] Trudinger, N.S. On the twice differentiability of viscosity solutions of nonlinear elliptic equations, Bull. Aust. Math. Soc. 39(1989), 443-447.

[21] Caffarelli, L. Elliptic second order equations, Lecture Notes (1988).

Neil S. Trudinger

Centre for Mathematical Analysis

Australian National University

GPO Box 4 Canberra ACT 2601

AUSTRALIA 